\title{
Locus ceruleus neurons in people with autism contain no histochemically-detectable mercury
}

\author{
Roger Pamphlett • Stephen Kum Jew
}

Received: 20 November 2015/Accepted: 21 November 2015/Published online: 27 November 2015

(C) The Author(s) 2015. This article is published with open access at Springerlink.com

\begin{abstract}
Exposure to environmental mercury has been proposed to play a part in autism. Mercury is selectively taken up by the human locus ceruleus, a region of the brain that has been implicated in autism. We therefore looked for the presence of mercury in the locus ceruleus of people who had autism, using the histochemical technique of autometallography which can detect nanogram amounts of mercury in tissues. In addition, we sought evidence of damage to locus ceruleus neurons in autism by immunostaining for hyperphosphorylated tau. No mercury was found in any neurons of the locus ceruleus of 6 individuals with autism (5 male, 1 female, age range 16-48 years). Mercury was present in locus ceruleus neurons in 7 of 11 (64\%) age-matched control individuals who did not have autism, which is significantly more than in individuals with autism. No increase in numbers of locus ceruleus neurons containing hyperphosphorylated tau was detected in people with autism. In conclusion, most people with autism have not been exposed early in life to quantities of mercury large enough to be found later in adult locus ceruleus neurons. Human locus ceruleus neurons are sensitive indicators of mercury exposure, and mercury appears to remain in these neurons indefinitely, so these findings do not
\end{abstract}

R. Pamphlett $(\bowtie) \cdot$ S. Kum Jew

Discipline of Pathology, The University of Sydney, Brain and Mind Centre, Camperdown, NSW 2050, Australia e-mail: roger.pamphlett@sydney.edu.au

S. Kum Jew

e-mail: stephen.jew@sydney.edu.au support the hypothesis that mercury neurotoxicity plays a role in autism.

Keywords Autism · Mercury - Locus ceruleus · Locus coeruleus $\cdot$ Toxicant $\cdot$ Heavy metal

\section{Introduction}

Environmental toxins (i.e., toxicants) have long been suspected to play a role in the pathogenesis of autism, but there is still disagreement as to what toxicants, if any, could be involved (Lyall et al. 2014). One toxicant that has been repeatedly implicated in autism is mercury (Kern et al. 2012; Mutter et al. 2005). Although some autism studies have reported environmental exposures to mercury during early life, and measured various biomarkers of mercury exposure, no consensus as to the role of mercury in autism has been reached. One area of research in this field where data are notably lacking is in assessments of toxic metals such as mercury in the brains of people with autism.

The region of the human brain that takes up most mercury is the locus ceruleus in the pons (Pamphlett and Kum Jew 2013b), probably because these neurons innervate most of the blood vessels in the brain and so are exposed to circulating toxins (Pamphlett 2014). Once mercury enters locus ceruleus neurons it appears to remain there indefinitely (Pamphlett and Kum Jew 
2015), which suggests the locus ceruleus can act as a indicator of past exposure to this toxic metal. Human exposure to mercury appears to be quite common, since 43 out of $60(72 \%)$ adults without neurological or psychiatric disorders have been shown to have mercury within locus ceruleus neurons (Pamphlett and Kum Jew 2013a; 2015). A decrease of noradrenaline output from locus ceruleus neurons has been postulated to underlie some of the features of autism (Martchek et al. 2006; Mehler and Purpura 2009), so it seems worthwhile to look for the presence of mercury in the locus ceruleus in people who have had autism.

A histochemical technique that can detect nanogram amounts of mercury in tissues is autometallography (Danscher and Moller-Madsen 1985), so we used this method to look for evidence of mercury in locus ceruleus neurons in people with autism compared to controls. We also looked for evidence of damage to locus ceruleus neurons in autism by staining for hyperphosphorylated tau (Braak and Del Tredici 2011).

\section{Methods}

Cases and controls

$7 \mu \mathrm{m}$ paraffin sections of pons containing the locus ceruleus were available from 6 individuals with autism from the Oxford Brain Bank (5 male, 1 female, mean age 27 years, age range 16-48 years). The diagnosis of autism was made during life on Autism Diagnostic Interview-Revised criteria. Causes of death were three cases of sudden unexpected death in epilepsy and one each of cystic fibrosis, glioblastoma multiforme, and water intoxication. Control stained sections of pons were available from 5 neurologically-normal controls (3 male, 2 female, age range 26-47 years) and 6 patients with amyotrophic lateral sclerosis (5 male, 1 female, age range 38-49 years) from previous studies of locus ceruleus neurons (Pamphlett and Kum Jew 2013a, 2015).

\section{Autometallographic staining for mercury}

Sections were stained with silver nitrate autometallography, which under routine conditions stains the sulphides or selenides of mercury, silver, and bismuth (Danscher and Moller-Madsen 1985; Danscher et al. 2000). The silver-coated deposits of these metals in tissues are seen histologically as black-staining grains, and referred to as $\mathrm{AMG}^{\mathrm{HM}}$ (autometallographydemonstrable heavy metals). Sections were placed in physical developer containing gum arabic, citrate buffer, hydroquinone and silver nitrate at $26{ }^{\circ} \mathrm{C}$ for $80 \mathrm{~min}$ in the dark, then washed in sodium thiosulphate to remove unbound silver. Sections were counterstained with mercury-free haematoxylin and viewed under bright-field microscopic illumination at $600 \times$ magnification. A positive control section was included of mouse spinal motor neurons that contained mercury following intraperitoneal exposure to mercuric chloride (Pamphlett and Png 1998). The percentage of locus ceruleus neurons containing $\mathrm{AMG}^{\mathrm{HM}}$ was calculated as previously described (Pamphlett and Kum Jew 2015).

AT8 staining for hyperphosphorylated tau

Paraffin sections were treated with hydrogen peroxide followed by normal horse serum and incubated in the monoclonal antibody AT8 AntiHuman Phos PHF Tau pSer202/Thr 205 (Thermo MN1020) at 1:1000 for $60 \mathrm{~min}$ at $37{ }^{\circ} \mathrm{C}$. Slides were incubated with Envision Dual Link HRP and visualized with 3,3 diaminobenzidine tetrahydrochloride, then counterstained with haematoxylin and viewed with bright-field microscopy.

\section{Ethics}

Sections of post mortem paraffin-embedded pons were obtained after application to the UK Oxford Brain Bank, which with approval from a research ethics committee collects and distributes consented donated brain tissue for research. No patient consent specifically for this project was therefore applicable. The work was carried out in accordance with the ethical standards of the Human Ethics Review Committee (RPAH Zone) of the Sydney Local Health District (X14-0029) and with the Declaration of Helsinki as revised in 2000. The authors declare they have no conflicts of interest.

\section{Results}

Autometallography

No $\mathrm{AMG}^{\mathrm{HM}}$ was seen in locus ceruleus neurons of any of the six individuals with autism (Fig. 1a). In 


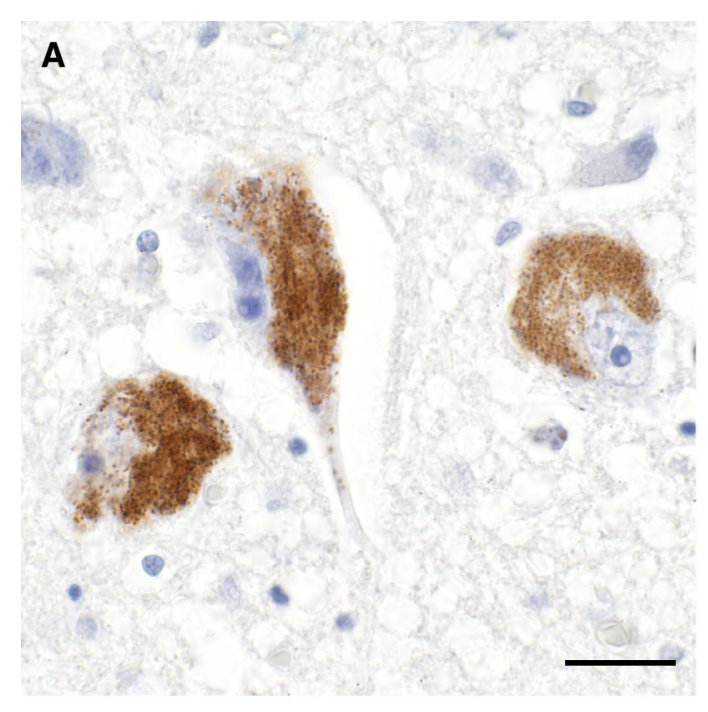

4Fig. 1 a Three locus ceruleus neurons in this individual with autism contain yellow-brown neuromelanin pigment, but no black grains of autometallographically-detectable mercury $\left(\mathrm{AMG}^{\mathrm{HM}}\right)$. b One locus ceruleus neuron in this patient with amyotrophic lateral sclerosis shows widespread (closed arrow), and one scattered (open arrow), $\mathrm{AMG}^{\mathrm{HM}}$; other neurons, including a non-pigmented neuron (arrow-head) show no $\mathrm{AMG}^{\mathrm{HM}}$. a, b Autometallography and hematoxylin. c Staining for hyperphosphorylated tau is seen in a locus ceruleus neurite (asterisks) in this individual with autism, but not in the cell body of an adjacent pigmented neuron. AT8 immunostaining and hematoxylin. All bars $20 \mu \mathrm{m}$
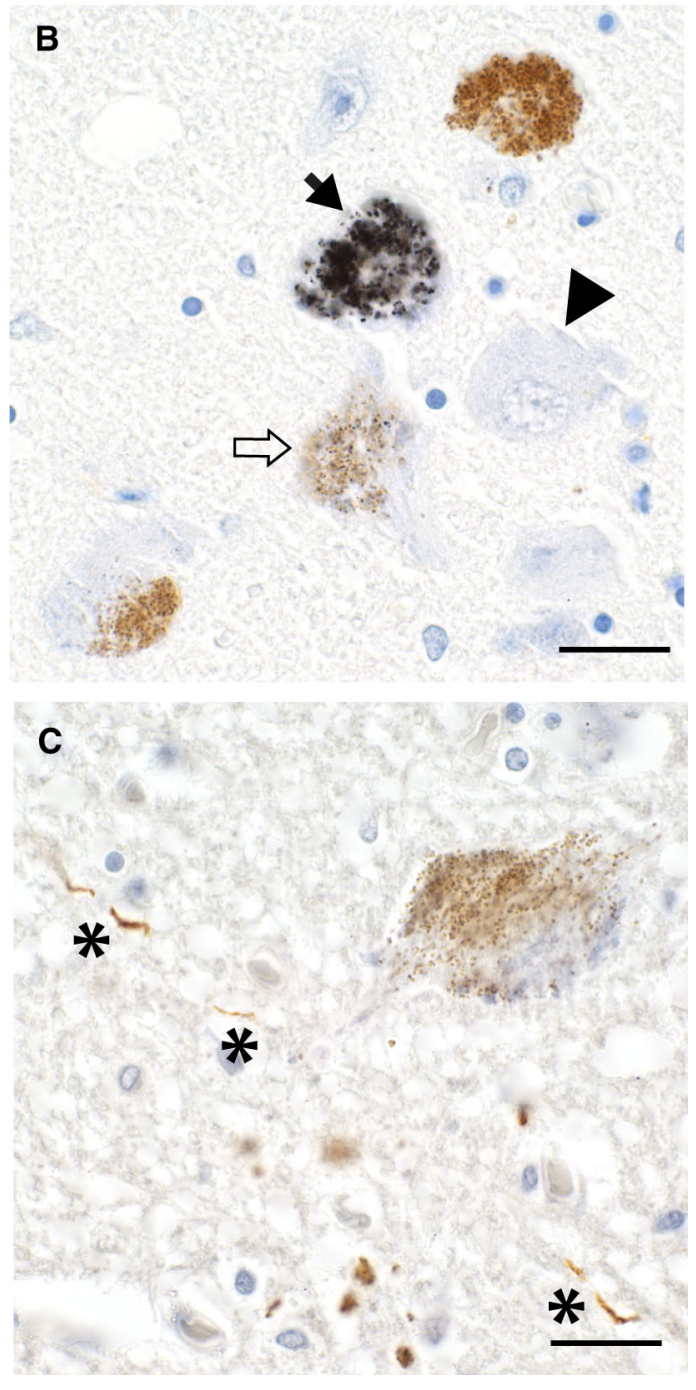

comparison with age-matched controls, using the same AMG technique, two of five neurologicallynormal controls had locus ceruleus neurons containing $\mathrm{AMG}^{\mathrm{HM}}$ (2 and $5 \%$ of neurons respectively), as did five of six amyotrophic lateral sclerosis patients $(4,5$, 17, 22 and $32 \%$ of neurons respectively) (Pamphlett and Kum Jew 2013a) (Fig. 1b). The presence of $\mathrm{AMG}^{\mathrm{HM}}$ in $\mathrm{LC}$ neurons of autism individuals was significantly less than for combined age-matched controls (Fisher's exact two-sided $\mathrm{p}=0.03$ ).

AT8 for hyperphosphorylated tau

AT8 staining was seen in one neurite in the locus ceruleus of one individual with autism (Fig. 1c), but in none of the other five individuals with autism. No cell bodies of locus ceruleus neurons of any autism individual showed AT8 staining. In comparison, using the same AT8 technique, in 12 individuals who had no neurological or psychiatric conditions (7 male, 5 female, age range 51-59 years) AT8 staining was present in locus ceruleus neurites in 3 individuals and in neuronal cell bodies in 2 individuals (Pamphlett and Kum Jew 2015).

\section{Discussion}

No mercury was detected in locus ceruleus neurons of people with autism using a sensitive histochemical technique that can show the presence of nanogram amounts of mercury. This is despite the locus ceruleus being the region of the human brain that takes up circulating mercury most avidly, and the frequent presence of mercury in these neurons in control individuals. Furthermore, no signs of damage to the 
locus ceruleus was seen on hyperphosphorylated tau immunostaining, beyond that expected for age. These findings do not support the hypothesis that exposure to mercury early in life is a risk factor for autism, or that the locus ceruleus is damaged in autism.

The study has a number of limitations. (1) Numbers of autism post mortem samples available for examination were limited, so it remains possible that a subset of people with autism could have mercury in their locus ceruleus neurons. Autism is seldom a lifethreatening disorder, so even brain banks that specialise in recruiting autism donors such as the one accessed here have only limited numbers of brain samples available for research. Nevertheless, statistically fewer people with autism had mercury in the locus ceruleus neurons than age-matched controls. (2) Trace amounts of mercury could have triggered an autoimmune response (Mutter et al. 2005) which destroyed the neurons that contained the mercury, so they would not have survived to be examined. There is however no evidence of a loss of locus ceruleus neuronal in autism (Martchek et al. 2006). (3) Mercury could transiently enter and then be cleared from locus ceruleus neurons (a "hit-and-run" effect). This seems unlikely, since neuromelanin binds heavy metals (Double et al. 2008) and the pigment appears already by the fifth month of gestation in locus ceruleus neurons (Foley and Baxter 1958). Mercury would therefore be expected to remain in these neurons indefinitely.

More work is needed to find out if a range of toxic element other than mercury could be present in the brains of people with autism. Multiple elements within cells can now be detected using synchrotron X-ray fluorescence microprobe analysis (Aitken et al. 2010). This technique could be used to look for a large number of toxic elements within locus ceruleus neurons, but this will depend on the availability of sufficient numbers of frozen pons samples from people with autism as well as controls.

In conclusion, our findings do not support the hypothesis that exposure to mercury is a risk factor for autism. The usual caveat of "absence of evidence is not evidence of absence" needs however to be heeded, and further efforts to analyse brain, and in particular locus ceruleus, toxins in autism should be encouraged.

Acknowledgments Paraffin sections were obtained from the Oxford Brain Bank, which is supported by the Medical Research
Council, Brains for Dementia Research, Alzheimer Society and Alzheimer Research UK, Autistica UK and the National Institute for Health Research Oxford Biomedical Research Centre. The project was supported by the Aimee Stacey Memorial and Ignacy Burnett Bequests.

Open Access This article is distributed under the terms of the Creative Commons Attribution 4.0 International License (http:// creativecommons.org/licenses/by/4.0/), which permits unrestricted use, distribution, and reproduction in any medium, provided you give appropriate credit to the original author(s) and the source, provide a link to the Creative Commons license, and indicate if changes were made.

\section{References}

Aitken JB, Carter EA, Eastgate H, Hackett MJ, Harris HH, Levina A, Lee YC, Chen CI, Lai B, Vogt S, Lay PA (2010) Biomedical applications of $\mathrm{X}$-ray absorption and vibrational spectroscopic microscopies in obtaining structural information from complex systems. Radiat Phys Chem 79:176-184

Braak H, Del Tredici K (2011) The pathological process underlying Alzheimer's disease in individuals under thirty. Acta Neuropathol 121:171-181

Danscher G, Moller-Madsen B (1985) Silver amplification of mercury sulfide and selenide: a histochemical method for light and electron microscopic localization of mercury in tissue. J Histochem Cytochem 33:219-228

Danscher G, Stoltenberg M, Kemp K, Pamphlett R (2000) Bismuth autometallography: protocol, specificity, and differentiation. J Histochem Cytochem 48:1503-1510

Double KL, Dedov VN, Fedorow H, Kettle E, Halliday GM, Garner B, Brunk UT (2008) The comparative biology of neuromelanin and lipofuscin in the human brain. Cell Mol Life Sci 65:1669-1682

Foley JM, Baxter D (1958) On the nature of pigment granules in the cells of the locus coeruleus and substantia nigra. J Neuropathol Exp Neurol 17:586-598

Kern JK, Geier DA, Audhya T, King PG, Sykes LK, Geier MR (2012) Evidence of parallels between mercury intoxication and the brain pathology in autism. Acta Neurobiol Exp 72:113-153

Lyall K, Schmidt RJ, Hertz-Picciotto L (2014) Maternal lifestyle and environmental risk factors for autism spectrum disorders. Int J Epidemiol 43:443-464

Martchek M, Thevarkunnel S, Bauman M, Blatt G, Kemper I (2006) Lack of evidence of neuropathology in the locus coeruleus in autism. Acta Neuropathol 111:497-499

Mehler MF, Purpura DP (2009) Autism, fever, epigenetics and the locus coeruleus. Brain Res Rev 59:388-392

Mutter J, Naumann J, Schneider R, Walach H, Haley B (2005) Mercury and autism: accelerating evidence? Neuroendocrinol Lett 26:439-446

Pamphlett R (2014) Uptake of environmental toxicants by the locus ceruleus: a potential trigger for neurodegenerative, demyelinating and psychiatric disorders. Med Hypotheses 82:97-104 
Pamphlett R, Kum Jew S (2013a) Heavy metals in locus ceruleus and motor neurons in motor neuron disease. Acta Neuropathol Commun 1:81

Pamphlett R, Kum Jew S (2013b) Uptake of inorganic mercury by human locus ceruleus and corticomotor neurons: implications for amyotrophic lateral sclerosis. Acta Neuropathol Commun 1:13

Pamphlett R, Kum Jew S (2015) Different populations of human locus ceruleus neurons contain heavy metals or hyperphosphorylated tau: implications for amyloid-beta and tau pathology in Alzheimer's disease. J Alzheimers Dis 45:437-447

Pamphlett R, Png FY (1998) Shrinkage of motor axons following systemic exposure to inorganic mercury. J Neuropathol Exp Neurol 57:360-366 